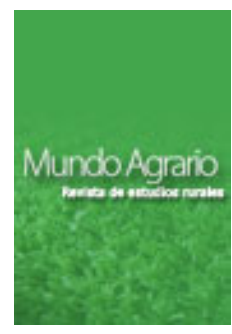

Mundo Agrario vol. 18, n 37, e043, abril 2017. ISSN 1515-5994

Universidad Nacional de La Plata.

Facultad de Humanidades y Ciencias de la Educación.

Centro de Historia Argentina y Americana

\title{
Coaliciones sociales y políticas en el desarrollo del sector agroexportador: las frutas murcianas y el trabajo en las redes globales de producción agroalimentaria
}

\author{
The role of social and political coalitions on the development of \\ the agro-export sector Coalitions: Murcian fruit and work in global \\ agri-food production networks
}

\section{Carlos de Castro *, Elena Gadea **, Andrés Pedreño *, Antonio J. Ramírez ** \\ * Universidad Autónoma de Madrid, ** Universidad de Murcia, España | c.decastro@uam.es, megadea@um.es, andrespe@uam.es, gastoni@linuxmail.org}

\section{PALABRAS CLAVE RESUMEN}

Industria agroalimentaria

Relaciones de trabajo

Acoplamiento estratégico

Redes globales de producción

\section{KEYWORDS}

Agri-food industry

Work

Strategic coupling

Global production networks
El territorio de la vega media del Segura en la Región de Murcia está históricamente articulado con cadenas globales de productos primarios (esparto) y agroalimentarios (frutas). Estas articulaciones históricas han operado a través de coaliciones de fuerzas sociales cambiantes en función del producto y en función del ciclo histórico. Concretamente, la producción agroalimentaria ha experimentado dos momentos que se presentan discontinuamente en el tiempo: el primer ciclo exportador hortofrutícola se inicia a comienzos del siglo XX y se cierra en los años 50 y tiene como protagonista a la industria conservera; y el segundo ciclo exportador hortofrutícola arranca a partir de los años 60 , se consolida a finales de los 80 y principios de los 90 , y perdura hasta la actualidad, protagonizando este segundo momento la producción de fruta para mercado en fresco. En este complejo escenario el trabajador asalariado ha tenido una enorme centralidad, aunque se ha presentado profundamente segmentado en una variedad de figuras productivas -el jornalero agrícola, el recolector de esparto, las mujeres de los almacenes de manipulado o de las conserveras, el trabajador de las fábricas de esparto, etc.- y en las cuales las líneas de diferenciación por clase, género, etnia e inclusive edad han tenido un enorme papel. El artículo pretende mostrar las dimensiones políticas del proceso histórico de construcción de este espacio productivo desde la perspectiva de una sociohistoria de las relaciones de trabajo en conexión con las formas cambiantes de articulación del territorio con las cadenas globales de mercancías. Para ello, este artículo se apoyará en las aportaciones de los estudios sobre las cadenas globales de mercancías y redes globales de producción, así como de los estudios regionales.

\section{ABSTRACT}

The territory of the Vega Media del Segura in Murcia is historically articulated to global commodity chains (Esparto) and agri-food (fruits). These historical articulations have operated through changing coalitions of social forces depending on the product and depending on the historical cycle. Specifically, food production has experienced two moments that occur discontinuously in time: the first fruit and vegetable export cycle begun in the early twentieth century and closed in the 50s; fruit and vegetable exporter. The second exportation cycle started from the 60s, consolidated in the late 80s and early 90s and lasts until today. In this complex scenario wage work has had an enormous centrality, although there has been deeply segmented into a variety of production figuresagricultural laborer, the collector Esparto, women stores manipulated or canneries, worker esparto factories, etc. and in which the lines of differentiation by class, gender, ethnicity and even age have had a huge role. The article shows the political dimensions of the historical process of the construction of this productive space from the perspective of a socio-history of labor relations in connection with the changing forms of articulation of territory with global commodity chains. To do this article will build on the contributions of studies on global commodity chains and global production networks and regional studies. 


\section{Introducción}

Al igual que otras regiones del sur de Europa, en el último siglo la Región de Murcia ha basado su desarrollo en la especialización productiva en sectores de bajo valor añadido, intensivos en mano de obra, dependientes de mercados externos y con unas relaciones de trabajo caracterizadas por una fuerte eventualidad. Estos sectores en el caso de Murcia han sido históricamente la agricultura comercial e intensiva y más tarde el turismo y la construcción.

La integración o acoplamiento del sector agroalimentario a las redes globales agroalimentarias ha sido una de las principales estrategias de desarrollo de la región y ha conllevado históricamente un profundo proceso de restructuración institucional, productiva y social en el que han colaborado numerosos actores económicos y políticos.

Estos procesos de conexión entre las dinámicas globales y las estrategias regionales de desarrollo se han estudiado de manera intensa desde los años 80 en diferentes campos disciplinares. Este artículo se apoyará en la literatura de las cadenas y las redes globales de producción (RGP) (Gereffi et al, 2005; Coe et al, 2008) y en la literatura de los estudios regionales (Pike et al, 2011, 2006; Martin, 2015). Ambas literaturas han analizado estos procesos de manera despolitizada y, cuando han intentado analizar las dimensiones políticas, lo han hecho desde una concepción de lo político muy restringida. En el caso de los enfoques de las cadenas y redes globales de producción han estudiado las disputas por la distribución del poder entre las empresas dentro de las RGP y entre las empresas y las instituciones regionales, mientras que los estudios regionales han priorizado el análisis de las disputas competenciales entre diferentes escalas de la administración pública.

Este artículo pretende ampliar la concepción de lo político en estos procesos. La idea principal consiste en subrayar la naturaleza política y disputada de este proceso de restructuración regional orientado a facilitar el acoplamiento estratégico de una región en las redes globales de producción agroalimentaria. Nuestra hipótesis es que la expansión del sector agroalimentario de la Región de Murcia ha estado impulsada por una compleja y conflictiva coalición de fuerzas sociales, políticas e institucionales, regionales y extrarregionales, que ha ido cambiando a lo largo del tiempo. Esto implica estudiar las múltiples dimensiones políticas que abarca ese proceso a escala regional, estatal y supraestatal.

Estas coaliciones sociales y políticas se encargan de impulsar los procesos de transformación productiva, institucional, legislativa, etc., que crean las condiciones que facilitan a los actores económicos el acceso a los recursos necesarios para acoplarse exitosamente en la red global de producción.

En efecto, el desarrollo de la industria agroalimentaria depende del acceso a un conjunto amplio de recursos: agua, tierra, trabajo, financiación, tecnología, redes comerciales, etc. La cuestión es que el acceso a estos recursos depende de que exista una coalición social de actores provenientes de las redes de poder corporativo local y extralocal, y de las redes de poder político local y extralocal. Una coalición que puede operar a varias escalas, que varía a lo largo del tiempo y que puede tener una composición diferente para cada uno de los recursos $\underline{1}$. Aquí se prestará atención a la coalición asociada al trabajo.

El territorio de la vega media del río Segura en la Región de Murcia es un buen ejemplo de ello. Esta comarca está históricamente articulada en torno a redes globales de productos primarios (esparto) y agroalimentarios (frutas y verduras). Estas articulaciones históricas han operado a través de coaliciones de fuerzas sociales cambiantes en función del producto y del ciclo histórico. Concretamente, la producción agroalimentaria ha experimentado dos momentos a lo largo del tiempo. El primer ciclo exportador hortofrutícola se inicia a principios del siglo XX y se cierra en los años 50 y 60 y tiene como protagonista a la industria conservera; y el segundo ciclo exportador hortofrutícola arranca a partir de los años 60, se consolida entre finales de los 80 y principios de los 90, y perdura hasta la actualidad; este segundo momento 
protagoniza la producción de fruta para mercado en fresco. El segundo ciclo exportador hortofrutícola ha experimentado profundos cambios desde los años 60 según lógicas de integración, especialización, intensificación, concentración de capital, etc. Al contrario que en la etapa anterior, en la que predominaban los pequeños productores, en el momento actual son grandes empresas de producción-comercialización de frutas en fresco las que dominan el territorio.

En este complejo escenario el trabajador asalariado ha tenido una enorme centralidad, aunque se ha presentado profundamente segmentado en una variedad de figuras productivas -el jornalero agrícola, el recolector de esparto, las mujeres de los almacenes de manipulado o de las conserveras, el trabajador de las fábricas de esparto, etc.-, en las cuales las líneas de diferenciación por clase y género han tenido un enorme papel.

Este artículo se centrará en analizar las dimensiones políticas de las estrategias de movilización del trabajo y de las coaliciones sociales que se han formado a su alrededor en cada una de las etapas del sector. La idea es reflejar la naturaleza política y disputada del proceso de construcción política del trabajo agrícola -que en cada etapa ha servido para que el sector pudiera acoplarse a las redes globales de producción agroalimentaria- centrándonos especialmente en el caso de la producción frutícola de Murcia.

A continuación, se muestra cómo varios enfoques han abordado la cuestión de la conexión entre las redes globales de la economía y las estrategias regionales de desarrollo. Y en la siguiente sección se describirán las dimensiones políticas de los procesos de construcción del espacio productivo regional y del mercado de trabajo a lo largo de las diversas etapas que ha atravesado el sector agroalimentario de la Región de Murcia desde finales del XIX hasta la actualidad $\stackrel{2}{ }$.

\section{Las dimensiones políticas del acoplamiento estratégico y de coaliciones sociales de crecimiento}

Resulta llamativa la escasa atención -salvo algunas excepciones (Levy, 2008; Glassman, 2011; Cumbers \& MacKinnon, 2011; Coe \& Hess; 2011)- que han recibido las dimensiones políticas de los procesos de construcción de las redes globales de producción (RGP) y de los procesos de reestructuración regional. Y es paradójico puesto que, como bien observa Glassman (2011), a partir del concepto de Cadenas de mercancías (CM) -en el que se inspira la idea de las RGP- Wallerstein y Hopkins pretendían realizar una reflexión política sobre las razones por las que los proyectos estatistas de los movimientos sociales de la izquierda histórica (socialdemócrata en el Norte Global, revolucionaria en el bloque del Este o nacionalista en el Sur Global) habían fracasado en su objetivo de "cambiar el mundo" tomando el control de los Estados. Wallerstein apunta que estos movimientos aprendieron la lección de que gran parte del poder se encuentra más allá de los límites del Estado; esto es, se puede estar en el gobierno y no tener el poder (Wallerstein, 2000). De ahí que propusiera el concepto de cadenas de mercancías como entidades difusas que cuestionan la centralidad del Estado y que producen un espacio económico global desigualmente desarrollado que supera la capacidad de control de los Estados $\underline{3}$ (Glassmann, 2011, p.155-6).

No obstante, esta sensibilidad política fue dejando paso a un análisis sobre la distribución del poder en el ámbito estrictamente empresarial. Gereffi (1994) retomó el concepto -denominado ahora cadenas globales de mercancías (CGM) - reorientándolo hacia el análisis de las estrategias y las acciones de las empresas que pueden impulsar dinámicas de desarrollo. Su idea era que la capacidad industrial y productiva no se creaba automáticamente por medio de las políticas comerciales de los Estados, sino también por medio de las estrategias de las empresas de competencia e innovación. De tal manera que Gereffi desplaza su atención, por un lado, hacia el análisis de la gobernanza de las cadenas globales de mercancías como una manera de captar las diferentes formas en que las empresas organizan y controlan las redes de producción transnacional, enfatizando el papel de las empresas líderes. Aquí Gereffi distinguió entre cadenas dirigidas por el productor 
y cadenas dirigidas por el comprador. Más tarde, haría una propuesta más compleja distinguiendo 5 formas de coordinación interempresarial: mercantil, modular, relacional, cautiva y jerárquica. (Gereffi 1994; Gereffi et al. 2005)

Y, por otro lado, desplaza su atención hacia las estrategias por medio de las cuales las empresas mantienen o mejoran su posición competitiva en la cadena (upgrading). También distingue varias estrategias de mejora (upgrading): funcional, de producto, de proceso e intercadenas (Gereffi et al. 2005).

Por otra parte, aunque Gereffi (1994) reconocía que el Estado y las instituciones pueden influir, acompañar o facilitar la formación de las cadenas globales de mercancías, considera al Estado y a las instituciones como algo externo e independiente de este proceso. Es una muestra clara de cómo se despolitiza el proceso de formación de un nuevo espacio económico otorgando un protagonismo nuclear a las empresas líderes.

El enfoque de las redes globales de producción (RGP) tratará de ampliar el marco para analizar el contexto institucional y social del territorio en el que se ubican las redes de producción (Henderson et al, 2002). Una red global de producción puede definirse como "un nexo globalmente organizado de funciones y operaciones interconectadas de empresas e instituciones no empresariales a través de las cuales se producen, se distribuyen y se consumen bienes y servicios” (Coe \& Hess, 2011, p. 130). Estos autores desplazan su atención, por tanto, hacia la conexión entre las redes globales de producción y el territorio y analizan las estrategias que la compleja red de actores (empresarios, asociaciones de empresarios, asociaciones ecologistas, trabajadores, sindicatos, asociaciones de consumidores, etc...) y las instituciones regionales despliegan para insertarse en la red global de producción (Coe \& Hess, 2011; 2013). Se trata de un análisis que contempla 3 variables. Por un lado, se analizan las estrategias de las empresas para crear valor (rentas tecnológicas, organizacionales, relacionales, de marca), capturar valor, así como las circunstancias bajo las cuales se facilita la creación de valor (tecnología, formación, conocimiento productivo del tejido empresarial local). Por otro lado, analizan cómo se distribuye el poder entre las empresas, las instituciones y la sociedad civil en varias escalas: estatal, regional y local. Y, por último, analizan la integración (embeddedness) de la RGP en el contexto institucional, cultural y social de la región. El análisis de esta última variable se ha profundizado por medio del concepto “acoplamiento estratégico” (Yeung, 2009) que se refiere a los procesos por medio de los cuales los diversos actores de las regiones se coordinan entre sí y establecen intereses estratégicos entre los actores locales y sus contrapartes globales (2009, p. 213).

El acoplamiento estratégico tiene 3 características. Es estratégico, en la medida en que necesita que participen de manera activa actores regionales y extrarregionales. Es contingente, en la medida en que implica la construcción de una coalición temporal que persiga un interés común entre grupos de actores que de otra manera no colaborarían entre sí. Y trasciende los límites territoriales en la medida en que interactúan actores de diferentes escalas espaciales (Coe y Hess 2011, p.132).

Esta ampliación del marco de análisis abre la puerta a considerar no sólo los procesos de negociación entre las empresas, y de las empresas con el Estado, como ocurría con el enfoque de las CGM, sino también el papel de otros actores: empresas regionales grandes y pequeñas, trabajadores, asociaciones de empresarios, de consumidores, de ecologistas, sindicatos, instituciones regionales y locales, etc.

A pesar del intento de superación del sesgo empreso-céntrico, este enfoque continúa tomando a la empresa como unidad organizativa de referencia en el proceso de creación de valor y reproduce una visión marcadamente economicista del proceso de formación de RGP y de su acoplamiento con las regiones. Además, en los análisis empíricos inspirados en este enfoque suele prestarse atención de manera casi exclusiva al papel de las empresas y al apoyo que éstas reciben de las instituciones (i.e. Chen, 2011; Zhu \& He, 2016). 
Por otra parte, desde el campo de los estudios regionales también se ha abordado la conexión entre la economía global y el desarrollo de la región (Pike et al, 2011; 2017; Martin, 2015). Al igual que en el enfoque de las cadenas globales de mercancías, los estudios regionales también se impulsaron con una fuerte intencionalidad política en los años 70, al estudiar las características de los territorios inmersos en dinámicas de desigualdad internacional desde la teoría del desarrollo desigual o desde la teoría de la dependencia (Cumbers \& MacKinnon, 2011, p. 249). La escasa sensibilidad hacia lo político ha predominado en este campo desde los años 80, salvo algunas excepciones representadas por la teoría de la máquina de crecimiento de Logan y Molotch (1987) y por la teoría de los regímenes urbanos de Stone (1989), las cuales proceden más bien de los estudios urbanos. Y ha sido muy recientemente que se han empezado a demandar estudios que enfaticen las dimensiones políticas en estos procesos. En este caso, los análisis de los estudios regionales han puesto el acento en la descripción del denso entramado institucional y social (algo que se ha denominado de distintas maneras: clústeres, distritos industriales, sistemas regionales de innovación) que facilita y pone en marcha la conexión de la región con la economía global en el marco de una estrategia de desarrollo. Si en los casos anteriores, lo político queda restringido a una disputa por la captura del valor entre las empresas, o bien como una disputa por la distribución del poder entre las instituciones regionales y las empresas de la RGP por controlar el espacio económico, en el caso de los estudios regionales lo político aparece vinculado a la relación conflictiva entre las diferentes escalas de la administración; esto se debe, principalmente, a los procesos de devolución o de reestructuración de competencias derivados de los procesos de reestructuración territorial de los Estados, o de los procesos de integración en entidades supraestatales, como la Unión Europea.

El problema de fondo que comparten las tres aproximaciones mencionadas es la concepción de Estado y, en general, de las instituciones políticas como entidades territoriales separadas de los mercados, en las que, además, la subordinación de las instituciones a la lógica mercantil y a los requerimientos de las redes globales de producción aparece como algo aproblemático y no conflictivo para los actores.

Inspirado en algunos esfuerzos por integrar las dimensiones políticas tanto en el campo de las RGP como en el de los estudios regionales (Levy, 2008; Glassman, 2011; Allen \& Cohcrane, 2007; Cumbers \& MacKinnon, 2011; Selwyn, Martin, 2015; Pike et al., 2017), este artículo sostiene que las redes globales de producción y las instituciones políticas de diferentes escalas se constituyen simultánea y mutuamente y dan lugar a un nuevo espacio que es al mismo tiempo económico y político. Este nuevo espacio es el resultado de un complejo y disputado proceso de construcción política impulsado por una amplia coalición de actores económicos, políticos y sociales que varía en cada región y que cambia a lo largo del tiempo.

Esto implica que también es preciso analizar el proceso de formación de alianzas entre los numerosos actores económicos, políticos y sociales. Logan y Motoch (1987), en el ámbito de los estudios urbanos, señalan que es el deseo y la expectativa de crecimiento lo que crea consenso entre la coalición de los actores que impulsan los proyectos de transformación urbana (coalición de crecimiento), más concretamente, consenso entre las élites. Por su parte, Levy (2008), quien se inspira en las aportaciones de Gramsci, señala que la construcción del consenso debe incorporar elementos económicos pero también elementos políticos y culturales. La construcción de un consenso requiere por tanto el establecimiento de una afinidad económica, política y cultural entre, por un lado, los actores impulsores de la reestructuración regional para acoplarse a las RGP y, por otro lado, entre los actores impulsores y resto de la población, lo que requiere conseguir la complicidad y el respaldo generalizado de la población, lo que hace necesario conectar con sus intereses económicos y con su sensibilidad política y cultural. Además, no hay que perder de vista que esta operación de construcción de un consenso amplio se realiza siempre en una situación de desigual correlación de fuerzas entre los actores. En resumen, el proceso de acoplamiento estratégico de la industria de una región a las redes globales de producción está ligado a un proceso de construcción política de un espacio multiescalar (nueva regionalidad), que depende de la formación de alianzas sociales y políticas entre los actores económicos y 
políticos de la región y los actores económicos y políticos de la red global de producción y de las instituciones supraestatales. Este es un proceso multiescalar y políticamente disputado que se encuentra inserto en la estrategia de desarrollo de la región. En nuestro caso, el desarrollo del sector agroalimentario en la Región de Murcia ha atravesado varias etapas y ha estado impulsado por una coalición social variable de actores empresariales y políticos.

En lo que respecta al proceso de acoplamiento de los trabajadores de la región a las sucesivas redes globales de producción agroalimentaria, hay que tener en cuenta la configuración institucional (legislación laboral) y social (patriarcado, paternalismo) del trabajo, las estrategias empresariales e institucionales de movilización y reclutamiento laboral, y los diferentes tipos de conflictos que han planteado los trabajadores en las sucesivas etapas de desarrollo de este sector.

\section{El caso de la producción agroalimentaria en la Región de Murcia}

\section{1. “Abarán-Paris-Londres”. Identidad local en el primer ciclo de desarrollo frutícola (finales siglo XIX-1960s)}

Este ciclo está marcado por iniciativas de empresarios pioneros, escasa coordinación institucional, y una política signada por una organización patriarcal y paternalista del trabajo, a todo lo cual, en los años 40 -con el inicio de la dictadura franquista en 1939- se añadirá la represión y el control total de la vida política.

El escritor y pensador G. K. Chesterton, en sus reflexiones de principios del siglo XX sobre el largo proceso de modernización capitalista experimentando por Gran Bretaña constataba con melancolía y con profunda agudeza crítica que "la verdad es que la idea de que la pequeña propiedad evoluciona hacia el capitalismo es un retrato exacto de lo que prácticamente no sucede nunca", lo que le llevaba a concluir que "el capitalismo es un monstruo que crece en los desiertos”. Esta afirmación de Chesterton es también una formulación precisa de la forma de proceder del desarrollo de la hortofruticultura intensiva en la Región de Murcia, y particularmente en la Vega Alta del Segura. Pues efectivamente el complejo agroexportador de frutas no se desarrolló a partir de la pequeña producción de las huertas radicadas en la vega del río Segura, sino que requirió de una ruptura con los límites que constreñían el regadío al perímetro regado por las aguas del río. Como han planteado Segura y Pedreño (2006) "ante las dificultades que encuentra el sistema (de hortofruticultura intensiva) para expandirse y constituir unidades productivas de dimensión elevada en las áreas irrigadas y ocupadas por la hortofruticultura tradicional, debido a la escasez, fragmentación y alto precio del suelo, el crecimiento se proyecta sobre espacios exteriores a las mismas, constituidas por tierras de secano, monte, etc., de las que existe una gran disponibilidad” (2006, pp. 379-380). Esta discontinuidad o ruptura se hace progresivamente a lo largo del siglo XX sobre la base de tecnologías hidráulicas de cada vez mayor envergadura y complejidad que permiten trasformar al regadío los antiguos secanos e incluso el monte bajo (en un proceso continuo y sistemático que perdura hasta hoy). Efectivamente, la producción hortofrutícola intensiva se desplegó a partir de una lógica de "conquista del desierto", de tal forma que las viejas huertas tradicionales fueron quedando relegadas y marginalizadas conforme las explotaciones más racionalizadas y dimensionadas (según el imperativo de las economías de escala y la organización productiva capitalista) crecían en los nuevos regadíos ganados al "desierto”노․

En la Vega Alta del Segura se desarrolla tempranamente lo que historiadoras como María Teresa Pérez Picazo denominaron un primer ciclo de agricultura comercial de vocación exportadora en torno a la producción frutícola y de uva de mesa desde finales del siglo XIX y las primeras décadas del siglo XX. Se produce en este momento una ruptura primigenia de la frontera del perímetro de las vegas tradicionales que restringía la producción frutícola para acceder, a través de diversos ingenios hidráulicos, a expandir el perímetro del regadío hacia nuevas tierras cada vez más alejadas de la vega del río aunque, en sus inicios, 
tuvieran cierta continuidad. Estos sucesivos y continuos "saltos" en la expansión de superficies irrigadas se hacen en un primer momento sobre tecnologías hidráulicas de pequeña envergadura impulsadas por pequeños propietarios, "bien por medio de pequeños retoques a la infraestructura existente (alargamiento de las acequias del Horno y Charco en Cieza, etc.), bien instalando artefactos elevatorios que permitan extender lateralmente el dominio del regadío" (Pérez Picazo \& Lemeunier, 1990, pp. 172-173). Para 1931 prolifera la instalación de diversos artefactos elevadores a motor como el Motor San Pedro en Cieza, el motor Resurrección en Abarán o el Motor Progreso en Blanca (Gómez Espín, 2012). Se trataba de pequeños proyectos impulsados y llevados a cabo por pequeños empresarios o por la agrupación de pequeños empresarios.

El apoyo institucional, especialmente por parte del Estado, no vendría hasta más adelante con la construcción de la red de embalses y del trasvase Tajo-Segura y constituiría el impulso fundamental de la segunda etapa. A finales de los años 50 se concluiría la red de embalses de la cuenca del río Segura, la cual formaba parte de un megaproyecto estatal iniciado en 1926, con la creación de la Confederación Hidrográfica Nacional, que tenía el fin de regular los recursos hídricos de todas las cuencas hidrográficas por medio de la construcción de embalses de retención (Pérez Picazo \& Lemeunier, 1990, p. 233; Martínez Carrión, 2002, pp. 488-494). Será a partir de los años 70, con la proliferación de pozos para la extracción mediante motor de aguas subterráneas, y con la construcción del trasvase Tajo-Segura por parte del Estado, cuando se culminará el largo proceso de transformación al regadío intensivo de secanos y montes bajos.

\section{El despegue del sector: organización productiva, poder y control de los recursos de la comunidad local}

Este primer ciclo frutícola se despliega a partir de la vinculación entre la producción y el espacio local (que coincide con su delimitación administrativa como municipio). Es en la localidad donde arraiga una determinada coalición de crecimiento (Logan \& Molotch, 1987) o alianza estratégica de actores políticos, económicos y sociales que construyen "un espacio contiguo que posee el carácter de una entidad definida por cualidades especiales” (según la definición de localidad propuesta por Harvey, 2007, p. 243). De tal forma que la localidad deviene en estratégica a la hora de construir el despliegue de la fruticultura intensiva en la medida que dota de "coherencia estructural o territorial" a los procesos operativos del modelo de desarrollo (producción y consumo, oferta y demanda de mercancías y fuerza de trabajo, el conflicto y la acumulación, la cultura y el estilo de vida) y se convierte en elemento fundamental para la formación de conciencia e identidad local y para la subjetividad política ${ }^{\underline{5}}$. A continuación destacaremos algunos de los agentes y procesos de la coalición de crecimiento que posibilitaron el primer ciclo de producción frutícola en la vega alta del río Segura.

En primer lugar hay que destacar que el impulso del primer ciclo frutícola vino de la mano de orientaciones destinadas a la conserva, esto es, las denominadas frutas de hueso (albaricoque, ciruela, etc.) y naranjas; orientaciones que fueron impulsadas de manera individual por algunos de los pioneros del sector. Ya en las décadas fordistas de los años 50 y 60, la demanda de los países europeos de alimentos no perecederos posibilitaría un segundo ciclo de expansión frutícola por el salto de la industria conservera a economías de escala, de tal forma que una nueva norma de producción vehicula el paso de una fase artesanal a una fase agroindustrial protagonizada por la gran fábrica y la centralización/concentración de capital (Martínez Carrión, 2002).

Este largo ciclo frutícola está vinculado fundamentalmente a la industria conservera. En Abarán se localiza la primera fábrica de conservas de fruta en el año 1899, cuyo titular es Nicolás Gómez Tornero, y a partir de ese momento conoce una notable expansión en las primeras décadas del siglo XX. No obstante, en estas primeras etapas el sector se organiza principalmente con pequeños y medianos productores de capital autóctono, con una elevada presencia de cooperativas y con una baja inversión tecnológica. 
Por otra parte, en Abarán se acuñará el lema “Abarán, París, Londres” para resaltar la vocación exportadora de sus producciones; una localidad que en los años '60 emerge como un emporio industrial proletarizado en torno a las fábricas conserveras, y que conforma el "contexto social y el marco territorial en los que se insertan las unidades de producción, y sus procesos de trabajo configuran una estrecha red, ordenada y simbólica de la vida no laboral y dan lugar a lo que se identifica como conjunto poblacional coherente ${ }^{6}$, (Bouvier, 1986, p. 22; Palenzuela, 2014, p. 69)

De hecho, las localidades de nuestra área de estudio experimentarán una identificación con la cultura del trabajo de la industria conservera ${ }^{\underline{7}}$, lo que aportará un amplio conjunto de conocimientos compartidos, transmitidos y aprendidos de manera informal por la población de la comarca.

En segundo lugar, hay que subrayar que el despliegue desde fines del siglo XIX de la economía frutícola y agroexportadora de la Vega Alta del segura está ligado a la historia de cómo las nuevas élites agrarias propietarias y empresariales controlaron los recursos de la comunidad local y los adaptaron a la nueva lógica productiva emergente. Si revisamos la historia de los alcaldes de un municipio como Abarán a lo largo del siglo XX observaremos que en su mayor parte proceden de las élites locales agroexportadoras. Estas mismas élites en su estrategia de control de los recursos públicos y sociales también realizaron numerosas inversiones en los municipios, en los más diversos ámbitos (asistencial, infraestructuras, espacios culturales o de ocio, etc.), según una lógica de paternalismo patronal con el que moldear y disciplinar a la comunidad local (Sierra, 1990). De tal forma que la nueva clase agroexportadora pasará a la historia local como los impulsores benefactores de una modernización desde arriba. Por ejemplo, así es recordado entre los cronistas locales uno de los empresarios pioneros de la conserva y exportación de fruta en el Abarán de principios del siglo XX:

Nicolás era un hombre con gran 'visión empresarial' y, al mismo tiempo, con una gran 'sensibilidad social', y ésta en dos aspectos: en el ámbito laboral, es decir, dentro de su empresa; y en el ámbito municipal, o sea, en el contexto de su pueblo. En relación con su aspecto empresarial, él empezó llevando fruta a Madrid en bestias de carga, continuó como encargado de la fábrica de conservas Champagne Fréres Limited, hasta que se estableció por su cuenta en el negocio de la fruta, tanto en conserva como en fresco, comenzando así a amasar su fortuna. Pero no se limitó a lo agrícola sino que diversificó su campo de acción y así supo invertir en el ámbito inmobiliario tanto en Madrid como en Murcia, haciéndose con edificios o solares en lugares estratégicos. Vivía gran parte del año en el Hotel Palace de Madrid, donde recibía en el vestíbulo a gran cantidad de gentes que iban a proponerle negocios. Tuvo una gran amistad con D. Juan March que le propuso hacerse su socio, pero Nicolás rehusó para poder, según él, seguir conservando la amistad. En relación con su sensibilidad social, en el ámbito de su empresa, lo fundamental para él era que a sus obreros no les faltara el trabajo y por ello no paraba la faena, aunque él perdiera dinero. Por lo que respecta a su pueblo, intervino con su capital en empresas tan importantes para el bienestar de Abarán como la Central Eléctrica, la red de abastecimiento de aguas y construyó a sus expensas el Asilo y la ermita de los Santos Médicos” (Carrasco Molina, 2008) $\stackrel{\underline{8}}{\text {. }}$

\section{Una organización patriarcal del trabajo}

Desde los primeros desarrollos del ciclo frutícola en la Vega Alta del río Segura ha sido importante la movilización y el reclutamiento de trabajadores. Durante esta etapa, las necesidades de mano de obra se han cubierto de manera casi exclusiva con trabajadores de la comarca.

Este sector se organiza en torno a una serie de pequeñas y medianas empresas de carácter familiar, con una regulación de tipo paternalista, con un bajo nivel de mecanización de los procesos y un uso intensivo y estacional de la fuerza de trabajo. Un contexto muy propicio para la formación paulatina de una línea de 
segmentación sexual del mercado de trabajo agrario.

Valga la estampa que ofrece Castaño Molina (2012) sobre la división del trabajo por género en el Abarán de las primeras décadas del siglo XX, tanto en la actividad de la recolección de la fruta como en la de la industria conservera -en muchos aspectos una protoindustria-:

En la fábrica de conserva y fruta las condiciones eran similares en muchos aspectos, parte del trabajo se realizaba en la calle, la fábrica no tenía suficiente espacio y la vía no era transitable. Normalmente, los hombres realizaban sus labores en el campo y unos pocos en la fábrica; las mujeres que iban al campo solían ser "mozas de servicio" que trabajaban por las tardes en plena faena de verano. Dentro de la fábrica ayudaban unos 6-8 hombres, los cuales se encargaban de lo más pesado y soldaban a mano la tapa de la conserva "como un sombrero" con cobre y estaño. Sobre las mujeres habla de centenares, alrededor de 300-350. Fuera de la fábrica, en el campo, son en mayor número los hombres, aunque también trabajaban mujeres que macheaban la fruta.

La feminización del trabajo del sector agroalimentario en este primer ciclo es el resultado de un complejo proceso social y político en un contexto muy particular. Por un lado, debido al limitado tamaño de las fincas, para realizar el trabajo de recolección se recurría al grupo familiar extenso y ocasionalmente a trabajadores que procedían de las redes sociales comarcales.

Por otro lado, serán inicialmente las mujeres locales procedentes de familias campesinas las que se ocuparían en la industria conservera. El trabajo estacional y discontinuo de las mujeres en la industria conservera responde a una estrategia de pluriactividad de la unidad familiar, como forma de captar rentas suplementarias. Por tanto, la posición de estas mujeres en el mercado de trabajo agroalimentario se construyó como una continuidad de su posición en el orden agrícola tradicional, que vinculaba a la mujer a lo doméstico, con una presencia discontinua en el ámbito productivo, donde el trabajo se construye como ayuda (Narotzky, 1988). Una mano de obra que, construida socialmente en función de la categoría de género, definía en términos de "ayuda familiar" el valor de su fuerza de trabajo, con toda la estructura de percepciones y clasificaciones que ello implica: la consideración de que el lugar "natural” de la mujer es el hogar, y de que cuando trabaje lo hará puntualmente para "ayudar" al cabeza de familia, sin desatender las tareas que son consideradas propias de su sexo, es decir, las relacionadas con el ámbito doméstico (Pedreño, Gadea \& García, 2013).

En el campo o en los almacenes, la mujer hace trabajo por horas estacional pero altamente flexible (en las épocas de mucha intensidad de trabajo puede llegar a "velar" horas de trabajo nocturnas), pero, al mismo tiempo, también está al frente de su hogar. Su posición subalterna se desprende de esta regulación hogartrabajo. Por tanto, la organización familiar y comunitaria favorecía la constitución de un ejército de reserva femenino, altamente funcional a las necesidades de las empresas, y un mercado de trabajo segmentado sexualmente. Esta estampa de recuerdos de la infancia de A. Gómez Sánchez es ilustrativa:

... ¿Y los cientos de mujeres anónimas que con su trabajo hicieron y han hecho posible aun hoy día la economía de nuestro pueblo en el sector del manipulado de frutas? Eran verdaderas legiones de mujeres de nuestro pueblo junto con otras que venían de otros pueblos vecinos de Cieza y Blanca, las que en la época de la uva y el albaricoque trabajaban afanosamente para llevar un jornal a casa. Por cierto, ¿no os dice nada la palabra “velar”? Mujer abaranera trabajadora, en el almacén, en la fábrica, o en su casa, y que cuando llegaba el momento, también sabía ser señora y estar en su lugar (Gómez Sánchez, 2002).

Por otra parte, esta posición subordinada de las mujeres en el ámbito rural se verá reforzada por el clima de represión política de los trabajadores y sindicatos, y por la restrictiva legislación laboral que se implementaría a partir de los años 40 en la dictadura franquista y que estaría en vigor hasta finales de los 
años 70. El franquismo instauró un modelo de gestión de las relaciones laborales de carácter corporativista que sostenía que era posible armonizar los intereses de empresarios y trabajadores, y que reprimía cualquier acto de desobediencia o cuestionamiento de las reglas establecidas. Durante los años 40 y 50, estas regulaciones legitimarían la discriminación salarial entre hombres y mujeres y mantendrían los salarios en niveles de subsistencia lo que, unido al racionamiento de los alimentos, conduciría a un escenario de empobrecimiento de la clase trabajadora (Manzanares 2006; Sola, 2014).

Así pues, la organización patriarcal y paternalista del trabajo propia del orden agrario tradicional y -desde los años 40- la legislación laboral franquista constituirían el marco político dentro del cual se reclutaría a los trabajadores de la comarca y que, a su vez, prácticamente garantizaría la inexistencia durante décadas de conflictos laborales a pesar de las imposiciones de flexibilidad y contención salarial de las empresas agrícolas.

Por último, la falta de alternativas laborales en estos territorios también jugará a favor de la feminización de la fuerza de trabajo. Esta zona se ha caracterizado históricamente por una actividad agrícola insuficiente, que hace que los trabajadores tengan que poner en marcha estrategias de movilidad sectorial y territorial. En época de recolección, los hombres solían buscar trabajo en otras regiones (Andalucía, principalmente). Para las mujeres, responsables de la reproducción de los grupos domésticos, la movilidad no siempre era una opción, y la cercanía de la actividad conservera y de manipulado compensaba las peores condiciones laborales al permitirles compatibilizar el trabajo dentro y fuera del hogar.

\section{2. "La huerta de Europa”. La construcción de la regionalidad en el segundo ciclo de desarrollo frutícola (1960s-actualidad)}

El segundo ciclo agroexportador arranca en los años 60 y se consolida en los 80. La apertura económica de la dictadura franquista en los años 60 permitió al sector fortalecer sus conexiones internacionales. En los 70 las empresas empezaron a aumentar su tamaño gracias, en parte, al acceso a la ampliación del perímetro de regadío, y empezaron también a aumentar su productividad gracias a una mayor inversión en la mecanización de los procesos.

La integración en la UE en 1986 supondrá el espaldarazo definitivo para la expansión e internacionalización del sector y supuso la consolidación del proceso de constitución de Murcia como una región exportadora conectada ahora a las redes globales de producción agroalimentaria (McMichael, 2009; Camarero, en este monográfico).

Se produjo, por tanto, un desbordamiento de la escala local o comarcal, en la que se desarrollaba en la etapa previa la actividad productiva, y se desencadenó un intenso proceso de reestructuración regional dirigido a construir las condiciones que hicieran posible el acoplamiento del sector con la red global de producción agroalimentaria, en línea con la estrategia de desarrollo para el conjunto de la Región.

En otras palabras, se inició un proceso de construcción política de un nuevo espacio económico que, aunque coincidía con los márgenes administrativos de la Región de Murcia, se encontraba fuertemente conectado a las redes globales de la producción agroalimentaria. Un proceso de construcción política que se realizaría desde 1975 en un marco político diferente marcado por el proceso de la transición a la democracia.

Este cambio de escala en el sector o, como indica Keating (2004), "regionalización del territorio productivo", dio lugar a varios procesos: la obtención de una mayor cantidad de recursos productivos, un intenso proceso de reestructuración institucional, empresarial y productiva; una ampliación de la superficie cultivable, un aumento de las reservas de agua disponible para el riego, un aumento de la necesidad de mano de obra con un cierto grado de especialización, un aumento de la concentración empresarial, reconversiones productivas y varietales, innovaciones biotecnológicas, consolidación y apertura de nuevos canales comerciales 
internacionales, acceso a nuevas líneas de crédito o atracción de inversiones con las que financiar la expansión del sector, etc... Una tarea titánica que reclamaba, por un lado, la creación de nuevas instituciones públicas tanto estatales como regionales y locales y, por otro lado, la construcción de nuevas alianzas entre numerosos actores económicos y políticos, regionales y extrarregionales, así como el respaldo de la población.

\section{Construyendo un nuevo espacio económico regional: nuevos actores y nuevas alianzas}

Desde su constitución en autonomía en 1982 se impuso la necesidad de establecer un cierto grado de coherencia estructural entre las fuerzas sociales y económicas de la Región de Murcia. Uno de los instrumentos para ello fue la elaboración de los Planes de Desarrollo de la Región de Murcia desde 1986 (Martínez Carrión, 2002). Los Planes Regionales incluían medidas que facilitaban a las empresas el acceso a los recursos y su conexión con las compañías líderes de la red global de producción agroalimentaria, lo que dio lugar a un intenso y complejo proceso de reestructuración regional por el que se han creado nuevas instituciones y asociaciones y en el que han participado directa o indirectamente numerosos actores políticos y económicos: grandes empresas exportadoras, pequeños productores, grandes cadenas de distribución (supermercados), trabajadores inmigrantes, intermediarios laborales como empresas de trabajo temporal (ETTs), asociaciones de productores y exportadores (APOEXPA $\stackrel{9}{9}$, PROEXPORT $\stackrel{10}{\underline{10}}$, FECOAM $\stackrel{11}{ }$ ), agencias de desarrollo (Instituto de Fomento, INFO), centros de investigación (Instituto Tecnológico de Uva de mesa (ITUM), Instituto Murciano de Investigación y Desarrollo Agrario y Alimentario (IMIDA), fondos de la Unión Europea, agencias privadas de certificación, entidades financieras privadas (Cajamurcia) o públicas (Instituto de Crédito Oficial, ICO), etc.

Por otra parte, esta reestructuración del espacio económico regional también ha traído consigo una reestructuración del tejido empresarial. En los últimos años se ha formado un denso entramado empresarial muy especializado en el que algunos han observado las típicas características de un clúster (MartínezCarrasco \& Martínez, 2011). Actualmente, el núcleo central del sector está compuesto por empresas de producción en fresco y por empresas de transformación al que se añaden industrias auxiliares y de servicios avanzados. Se estima que entre las empresas del sector hortofrutícola y las de la industria de transformación alimentaria hay cerca de 1.000 empresas (DIRCE, 2015), que conforman el núcleo duro del sector agroalimentario en Murcia. De éstas, cerca de 600 son empresas exportadoras (INFO, 2016, p. 2). Además, el tejido empresarial se encuentra muy estratificado. Por un lado, hay un pequeño grupo de grandes empresas que son, al mismo tiempo, productoras y comercializadoras, que tienen toda su producción orientada a las exportaciones, que están conectadas con los grandes distribuidores que dominan las redes globales de producción agroalimentaria, que concentran la mayor parte de la superficie cultivable, que emplean a la mayor parte de los trabajadores del sector, que forman parte de consorcios más amplios junto con Universidades, centros tecnológicos, etc. orientados a la investigación e innovación tecnológica y que han incorporado de manera intensa numerosos avances (Arcas et al, 2014, p. 189).

Por otro lado, se encuentra un gran número de pequeños productores conectados principalmente a las grandes empresas de la región. La enorme competencia del sector y la elevada exigencia de innovación tecnológica para el cumplimiento con los estándares de calidad imprimen una fuerte presión para invertir en innovación, que es difícilmente soportable financieramente a largo plazo para los pequeños productores. Esto ha generado, por un lado, la paulatina reducción de pequeños productores desde los años 60 (tabla 1) y un aumento del tamaño medio de las explotaciones (de 7,69 ha por explotación en 1999 a 12,37 ha en 2009), especialmente, en el de las explotaciones de más de 50 ha를 (de 107,6 ha por explotación en 1999 a 134,5 ha en 2009). Por otro lado, también ha generado un proceso de intensa creación de cooperativas (INFO, 2015) entre los pequeños productores que se han mantenido con el fin de aprovechar conjuntamente las instalaciones de transformación y con el fin de crear una plataforma común que permita realizar 
innovaciones tecnológicas en las tareas de cultivo, cosecha y envasado, en colaboración con las instituciones.

A pesar del esfuerzo de los pequeños productores, los sucesivos informes de las instituciones regionales no dejan de advertir que uno de los retos que debe afrontar el sector consiste en integrar la producción en estructuras de una dimensión más amplia que permita aumentar el poder de negociación frente a las grandes cadenas de distribución y que permita ampliar la escala de las innovaciones tecnológicas (INFO, 2015, 2016). Las instituciones regionales, cediendo a la presión de las asociaciones de grandes empresas (APOEXPA, PROEXPORT), parecen haber priorizado la ampliación de la escala del sector, lo que ha generado fuertes conflictos entre pequeños y grandes productores (i.e. sobre uso ilegítimo de agua o de instituciones tecnológicas) y ha debilitado la ya de por sí vulnerable posición de los pequeños.

Tabla 1. Superficie agraria utilizada en Región de Murcia, 1962-2009

\begin{tabular}{|l|c|c|c|c|c|c|}
\hline & $\mathbf{1 9 6 2}$ & $\mathbf{1 9 7 2}$ & $\mathbf{1 9 8 2}$ & $\mathbf{1 9 8 9}$ & $\mathbf{1 9 9 9}$ & $\mathbf{2 0 0 9}$ \\
\hline $\begin{array}{l}\text { SUPERFICIE AGRARIA } \\
\text { UTILIZADA (SAU) (Has.) }\end{array}$ & $\mathbf{4 4 5 . 2 0 1}$ & $\mathbf{5 9 1 . 6 6 7}$ & $\mathbf{5 7 6 . 5 5 1}$ & $\mathbf{5 3 4 . 9 7 1}$ & $\mathbf{4 5 7 . 0 3 2}$ & $\mathbf{3 9 4 . 5 3 8}$ \\
\hline Explotaciones con tierras & & & & & & \\
\hline < 0,5 Ha & 4.058 & 4.185 & 5.251 & 5.889 & 3.184 & 1.041 \\
\hline $\mathbf{0 , 5 - 1 ~ H a ~}$ & 8.327 & 7.486 & 8.483 & 8.115 & 5.603 & 2.144 \\
\hline $\mathbf{1 - 3}$ Ha & 25.022 & 26.264 & 29.427 & 29.741 & 21.218 & 14.595 \\
\hline 3-30 Ha & 185.956 & 186.790 & 181.756 & 168.453 & 131.034 & 109.045 \\
\hline $\mathbf{3 0 - 2 0 0 ~ H a ~}$ & 172.695 & 245.539 & 225.186 & 191.910 & 161.828 & 159.514 \\
\hline$>\mathbf{2 0 0}$ Ha & 49.143 & 121.403 & 126.449 & 130.863 & 134.166 & 108.198 \\
\hline
\end{tabular}

Fuente: INE. Censo Agrario. 2009 es el último año disponible.

Por otra parte, el tejido empresarial también está compuesto por una importante industria auxiliar y de servicios avanzados. Esta rama auxiliar del clúster agroalimentario estaría compuesta por empresas de transportes y de servicios logísticos especializados, de fabricación de envases, empresas de fitosanitarios, empresas de fabricación e instalación de invernaderos y otras estructuras de protección de cultivos, empresas de artes gráficas, empresas de suministro de aguas, sistemas de fertirrigación y sistemas de riego, empresas de fabricación de maquinaria agrícola e industrial, viveros (semillas, plantones, substratos), empresas de gestión y tratamiento de residuos, empresas de certificación de calidad, ETT's, etc. La creciente importancia del sector a nivel global ha provocado que algunos de estos subsectores se hayan convertido en una referencia en el mercado global y que también se hayan convertido en exportadores de tecnología productiva (Martínez-Carrasco \& Martínez, 2011, p. 186, INFO 2016, p. 6).

El caso de la empresa Frutas Esther, radicada en el municipio de Abarán, ejemplifica la evolución reciente del sector. Actualmente esta empresa es una de las más importantes en volumen de producción, exportación y de empleados de toda la Región de Murcia. Se encuentra integrada en varias asociaciones de productores y consorcios de investigación (Instituto Tecnológico de la Uva de Mesa, (ITUM), etc.) y es un actor influyente en las alianzas locales y regionales.

Frutas Esther también sirve para mostrar cómo se han redefinido las alianzas sociales y políticas que impulsan el desarrollo del sector. En el primer ciclo, bastaba con que las empresas trabaran alianzas con los ayuntamientos de los municipios de la comarca. De hecho, el fundador de Frutas Esther llegó a ser alcalde de Abarán. Sin embargo, en el nuevo ciclo de internacionalización del sector, las alianzas debían regionalizarse 
hasta alcanzar cierta influencia en las instituciones autonómicas e incluso estatales $\underline{13}$.

El papel de las asociaciones empresariales del sector como grupo de presión ante el Ministerio de Industria, de Agricultura, ante las consejerías ha sido decisivo puesto que ha logrado que numerosas instituciones incorporen a su agenda algunas de sus demandas más importantes. Por ejemplo, han conseguido implicar a varias instituciones en la celebración de numerosas ferias comerciales del sector (Fruit Atraction), en la inclusión de algunos temas en la agenda de negociación de la Política Agraria Común (PAC), han promovido movilizaciones por un nuevo trasvase Ebro-Segura,...

Esta reconstrucción regional de las alianzas muestra la naturaleza política y disputada del proceso de construcción del sector y su inserción a la red global agroalimentaria. Un proceso que, por otra parte, no ha estado exento de conflictos, sino que se trata de un proceso político disputado (Coe \& Hess, 2011). Los conflictos han girado en torno a diversos aspectos de la creación de las condiciones que permitan el acoplamiento de la región en las redes globales de producción agroalimentaria: conflictos derivados de la competencia entre las grandes empresas regionales y los pequeños productores, conflictos entre las empresas del sector y las instituciones por cambios institucionales y legislativos para acceder a algunos recursos $\underline{14}$, conflictos por la gestión del agua entre grandes productores y comunidades de regantes (Pedreño, Baños y Costantini, 2006) entre varias autonomías -Aragón y Murcia-, y varias administraciones públicas -Estado y Región de Murcia- a propósito del transvase Ebro-Segura $\frac{15}{}$, conflictos ecológicos entre representantes del sector y plataformas ecologistas por el deterioro del suelo y el despilfarro del agua $\frac{16}{}$, y conflictos laborales entre empresarios y trabajadores por las condiciones de trabajo del sector. Sobre este último, se ofrecen más detalles en el siguiente apartado.

\section{Organización flexible del trabajo y conflictividad laboral. La llegada de los trabajadores extranjeros}

En general, aunque a pesar de algunos altibajos el sector no ha dejado de crecer desde los años 70 , se ha producido un proceso de precarización de las condiciones de trabajo: estacionalidad, generalización de la contratación temporal, uso de intermediarios laborales, bajos salarios (de Castro, 2014).

Estas condiciones son el resultado de un disputado y conflictivo proceso de construcción política y social del mercado de trabajo agrícola. Lo más llamativo de este proceso ha sido la reproducción de posiciones vulnerables como necesidad estructural para acoplarse exitosamente en la red global de la producción agroalimentaria (Corrado, de Castro \& Perrotta, 2017), un proceso que no ha estado exento de conflictos (Pedreño, Gadea \& de Castro, 2014; de Castro, Moraes \& Cutillas, 2017).

En primer lugar, la construcción política del mercado de trabajo está vinculada a la constitución de un marco de legislación laboral que se desarrolló durante la transición a la democracia desde 1975 y que es equiparable a la del resto de países europeos, aunque se mantuvo vigente el Régimen Especial Agrario de la Seguridad Social que se desarrolló en 1971 y que establecía un trato discriminatorio respecto del resto de los trabajadores. De hecho, durante los años 70, y especialmente a partir de la llegada de la democracia, los sindicatos llevarían adelante una intensa labor para eliminar el Régimen Especial y elaborar regulaciones formales que equipararan las relaciones laborales a las de otros sectores industriales, y también para sustituir las regulaciones informales de tipo patriarcal que caracterizaron la primera etapa de la industria agroalimentaria.

En segundo lugar, la construcción política del mercado de trabajo está ligada al proceso de asalarización masiva de la mano de obra, debido a la estructuración del sector en torno a empresas de cada vez mayor tamaño, lo que propició el desarrollo de un movimiento sindical agrario en la Región. El sindicalismo agrario en la Región de Murcia se organizaba en esos años alrededor de la Federación de Trabajadores de la Tierra, de la Unión General de Trabajadores (U.G.T) y la Federación del Campo de Comisiones Obreras (CC.OO). 
Algunas de las reivindicaciones más importantes en las primeras negociaciones sindicales fueron el establecimiento del contrato fijo-discontinuo y la determinación del salario y la inclusión en el mismo de prestaciones que en otros sectores industriales estaban reconocidas, como el cobro de las vacaciones y de las pagas extraordinarias. El trabajo sin contrato y, por tanto, sin cotización para la Seguridad Social era una práctica muy extendida en el sector, que tradicionalmente ha presentado altos niveles de economía sumergida. Esto suponía que los trabajadores, en especial las mujeres, no tenían derecho a prestaciones sociales por enfermedad, desempleo o jubilación.

La mejora de las condiciones laborales y salariales en la agroindustria no se jugó únicamente en las mesas de negociación colectiva ni fue un proceso exento de desacuerdos. Los años 80 fueron el escenario de intensos procesos de conflictividad laboral marcados por huelgas en la industria conservera, contra el cierre de empresas, y en los campos y almacenes de la nueva agricultura intensiva, por la reivindicación del contrato fijo-discontinuo, por la obtención del derecho al seguro de desempleo y por la abolición del Régimen Especial Agrario de la Seguridad Social y la inclusión de los trabajadores agrícolas en el Régimen General de la Seguridad Social.

Con la negociación colectiva y con las movilizaciones, el sindicalismo trataba de influir sobre las formas de reclutamiento y, de este modo, contener las estrategias empresariales de sustitución de los trabajadores. Los empresarios, por su parte, asumieron que el nuevo contexto sociopolítico les obligaba a negociar con los representantes sindicales, aunque intentaron limitar los acuerdos de mejora de las condiciones laborales y salariales a los trabajadores fijos de manera que no se extendieran a los trabajadores eventuales, mayoritarios en el sector (Manzanares, 2006).

En tercer lugar, otro de los grandes hitos del proceso de construcción política del mercado de trabajo fue el reclutamiento de trabajadores extranjeros procedentes de los flujos migratorios iniciados en los 90 y que eclosionarían en los 2000. La posibilidad de recurrir a trabajadores extranjeros para sustituir a los trabajadores nacionales permitió a los empresarios contener y debilitar el movimiento sindical de la región. Estas estrategias de sustitución de trabajadores están amparadas por una legislación estatal de extranjería $\underline{17}$ que sitúa a los trabajadores extranjeros en una posición subordinada respecto de los trabajadores nacionales en la medida en que sólo se conceden permisos de trabajo si no hay suficientes trabajadores nacionales. Esta legislación, a su vez, vincula la estabilidad jurídica de los trabajadores extranjeros a su situación laboral, ya que la concesión de permisos de residencia está ligada a la posesión de un contrato laboral.

Tanto la legislación laboral como la legislación de extranjería, así como la propia estacionalidad del trabajo agrícola contribuyen a reforzar la vulnerabilidad de los trabajadores. Los empresarios han contribuido a reproducir esa posición vulnerable por medio de sus estrategias de sustitución de trabajadores, con el fin mejorar su posición competitiva en las redes globales de producción agroalimentaria.

Asimismo, los empresarios han tratado de contrarrestar las acciones sindicales de regulación y de frenar las demandas de los trabajadores desarrollando diversas estrategias como las de sustitución de trabajadores y la externalización del reclutamiento de la mano de obra. En un primer momento, en los años 70 y 80, los trabajadores del sector procedían de las bolsas tradicionales de jornaleros del Sur de España, que eran reclutados a través de procesos de movilidad itinerante y de migraciones internas. A mediados de los 80 , los trabajadores marroquíes empezaron a sustituir a las familias jornaleras locales. Durante los años noventa se consolidaron en ese mercado de trabajo y empezaron a realizar reivindicaciones laborales, demandas de regularización jurídica, y a participar intensamente en el movimiento sindical (Castellanos \& Pedreño, 2001). A finales de los años 90, los empresarios locales empezarían a sustituirlos por trabajadores ecuatorianos, cuyo número era creciente por causa del aumento de las migraciones internacionales, y esto fomentaba así la competencia con los trabajadores marroquíes y fortalecía la disciplina laboral. A principios de la década de los 2000 fueron los trabajadores ecuatorianos quienes participaron en los movimientos de "encierros de sin 
papeles” en diferentes partes de la Región de Murcia como protesta a la Ley de Extranjería y a las condiciones de trabajo del sector (Castellanos \& Pedreño, 2001).

Las estrategias de sustitución étnica no son las únicas que los empresarios locales del sector han utilizado para debilitar la posición de los trabajadores, sino que también se ha recurrido a varias formas de externalización del reclutamiento. Por un lado, está la elaboración de convenios de contratación en origen (Reigada, 2012; Hellio, 2016), más utilizadas en otras regiones y en otros cultivos y, por otro lado, está el recurso a los intermediarios laborales, una figura que se ha ido adaptando a los cambios en la configuración de la agricultura. En el sector agroalimentario en la Región de Murcia podemos distinguir dos momentos en la organización de la intermediación laboral: los años 90, con la expansión de los furgoneteros, y los años 2000, con el creciente protagonismo de las ETT (Castellanos \& Pedreño, 2001; Gadea, de Castro \& Pedreño, 2017).

Lo más importante de estas estrategias de externalización del reclutamiento no es tanto la reducción de costes sino el incremento del control social que permiten alcanzar en el seno de la relación laboral. No obstante cada estrategia empresarial de control es respondida por una nueva oleada de movilizaciones. El último caso de movilización es el de la formación de un sindicato agrario de inmigrantes en la Región de Murcia, Agrupación Laboral Autónoma de Fuerza Agraria (Alafa), que desde su creación en marzo de 2016 ha conseguido afiliar a más de 300 trabajadores en toda la Región (casi exclusivamente marroquíes) y organizar varias huelgas como medida de presión ante la negociación de convenios en varias empresas $\underline{18}$.

\section{Conclusiones}

Este artículo ha tratado de mostrar cómo el proceso de acoplamiento entre el sector agroalimentario de la Región de Murcia y las redes de la economía internacional ha estado impulsado a lo largo del tiempo por cambiantes coaliciones sociales y políticas de actores.

La primera etapa está marcada por un tejido empresarial compuesto de pequeños productores, por iniciativas de empresarios pioneros bien conectados con los representantes de las instituciones locales, por una escasa coordinación institucional, y por una política marcada por una organización patriarcal y paternalista del trabajo, a lo que, en los años 40, con el inicio de la dictadura franquista en 1939, se añadiría la represión y el control total de la vida política.

En la segunda etapa, especialmente a partir de los 80, el desarrollo del sector está impulsado por la colaboración entre las instituciones regionales y los empresarios regionales que ponen en marcha un intenso proceso de reestructuración productiva, institucional y social. La incorporación masiva de trabajadores extranjeros a un sector que crecía en volumen representa uno de los hitos fundamentales. En un contexto político más abierto, la dinámica de conflictos laborales (en torno a las mejoras salariales, a la inclusión en el régimen general o a la regularización de los inmigrantes irregulares) ha sido respondida por diversas estrategias empresariales como la sustitución de trabajadores o la externalización del reclutamiento.

En ambos casos este proceso de acoplamiento ha estado ligado a una redefinición del espacio económico y político. En la primera etapa se constituyó un espacio comarcal ligado a las redes comerciales europeas en el que fueron las élites locales agrarias las que tomaron la iniciativa. En la segunda etapa se produjo una intensa transformación del espacio, se construyó una región fuertemente conectada a las redes comerciales nacionales y europeas pero, sobre todo, a partir de los 80, a las redes globales de producción agroalimentaria. Un proceso que también conllevó la regionalización de la coalición social y política. Vemos por tanto cómo la red de poder oligárquico local y regional se extiende a lo largo del tiempo tejiendo alianzas entre empresas e instituciones locales y estatales. 
Al mostrar las diputas entre los actores en la constitución de estos espacios económicos hemos hecho visible la naturaleza política de la construcción del espacio económico y del mercado de trabajo regionales y de su conexión a las redes globales de la economía.

\section{Notas}

1 Aunque estén interrelacionadas, las coaliciones sociales pueden variar según se trate de trabajo, tecnología (clúster agroalimentario), agua, redes comerciales, suelo.

$\underline{2}$ La base empírica de este trabajo procede de diversas investigaciones que, desde hace más de una década, hemos venido desarrollando en la Región de Murcia sobre el empleo en la agricultura y las migraciones internacionales, utilizando fundamentalmente técnicas cualitativas.

3 Para una aclaración sobre el papel de Estado en el contexto global de las corporaciones transnacionales, véase Dicken (2011)

4 Sobre la metáfora del “desierto” en la modernización económica de la Región de Murcia, véase NdE han analizado la lógica económica de esta "conquista del desierto” para la expansión de la superficie del regadío intensivo, como un proceso en el que "la tierra -como factor de producción- experimenta una metamorfosis, pasando de su carácter originario e intrínseco de recurso natural apropiable, determinado por el grado de fertilidad intrínseca, al de “capital producido” por medio de intervenciones con carácter físico -creación artificial de suelo útil por medio de desmontes, roturaciones, abancalamientos, aportación de nutrientes, etc.y económico, ya que estas acciones son el resultado de la aplicación acumulativa de capital y de trabajo” (p. 380).

$\underline{5}$ Estamos siguiendo a Harvey (2007) en su propuesta de entender el espacio local o regional como lugar definido por los procesos operativos del desarrollo económico y dentro del cual se unen los mismos en una coherencia estructurada en una totalidad de fuerzas productivas y relaciones sociales.

6 Valga esta canción popular recogida por el cronista José S. Carrasco Molina que data, según sus propias apreciaciones, de las décadas de oro de la conserva (1950-1960), en la que se aprecia ese "conjunto poblacional coherente" según un juego de identificaciones entre el almacén frutícola, la localidad, su vida urbana, las mujeres abaraneras y las trabajadoras "naranjeras": A la entrada de Abarán/ Lo primero que se ve/ El almacén de Macanás/Y la casa del Chiqué/ Más abajo está el Cervantes/ Que es un gran Teatro/Con mucho valor/ Tiene un lujazo tremendo/ Y en el techo una araña/ Que vale un millón/.Viva Abarán, bonita población/Viva Abarán con su Calle Mayor/ Viva Abarán y las chicas abaraneras/ por donde quiera que vayan/ les llaman las naranjeras. (Recogida de una Charla de José S. Carrasco Molina sobre Abarán en sus mujeres, 11 de Mayo de 2006).

$\underline{7}$ Esta identificación ha sido señalada por la antropología de las culturas de trabajo: “cuando se produce una cierta concentración espacial de estos oficios, junto a una prolongada tradición productiva, se relacionan estas "culturas del trabajo" con la localidad que las acoge, convirtiéndose en un marcador de 'identidad local'” (Saglio, 1991; Palenzuela, 1995, p.18).

$\underline{8}$ http://josesimeoncarrasco.blogspot.com.es/2008/01/nicols-gmez-tornerouna-figura.html

$\underline{9}$ Asociación de Productores-Exportadores de Frutas, Uva de Mesa y Otros Productos Agrarios. http://www.apoexpa.es/

10 Asociación de Productores-Exportadores de Frutas y Hortalizas de la Región de Murcia. http://www.proexport.es/inicio/ 
$\underline{11}$ Federación de Cooperativas Agrarias de Murcia. http://www.fecoam.es/

12 http://www.ine.es/dyngs/INEbase/es/operacion.htm?

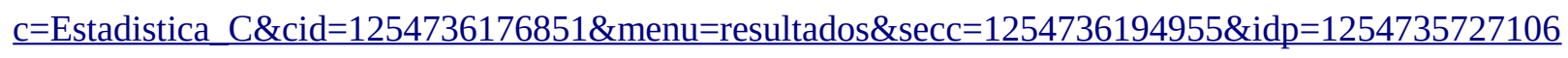

13 Información procedente de varias entrevistas a técnicos y gerentes de esta empresa así como de una visita a sus instalaciones en Abarán en el marco del Proyecto de I+D "Sostenibilidad Social de los Nuevos Enclaves Productivos Agrícolas” (ENCLAVES), 2011-2015.

14 Como ejemplo señalamos el testimonio del representante de APOEXPA a propósito de los problemas que genera el sector la falta de homogeneización de la regulación sanitaria entre la Unión Europea y otras instituciones: “...Yo os puedo contar un ejemplo que es de libro: una manzana o una ciruela tiene, cuando quieres exportarlo por barco que dura 30 días, tiene un tratamiento por cosecha antihongos, tú coges la ciruela, la terminas y la bañas en un producto y viaja de aquí a Brasil, que se exportan muchas ciruelas a Brasil. Bien, la Comunidad Europea prohíbe el tratamiento por cosecha en su país. Es decir, un español que va a exportar a Brasil mañana un contenedor de ciruelas no puede hacer ese tratamiento. La ciruela llega regular y entonces estamos muy limitados en la exportación de ciruela por ese tema. Pero la Organización Mundial del Comercio dice que el tratamiento por cosecha antihongos el mundo mundial tiene que autorizarlo y Europa importa sus ciruelas de Sudáfrica, por ejemplo, con el tratamiento por cosecha y calladito. Con lo cual, las ciruelas de Sudáfrica que tú ves en invierno, aunque no tengan sabor pero de aspecto son perfectas. Eso es algo que dices, y esto a quién se le ocurre...”

$\underline{15}$ Aquí puede verse un resumen; http://www.laopiniondemurcia.es/opinion/2014/03/26/agua/546610.html

16 La plataforma La región de Murcia no se vende ha criticado con frecuencia el deterioro medioambiental generado por diversas operaciones urbanísticas y por la ocupación del suelo protegido, y que está ligado a la agricultura así como a la propia industria agroalimentaria. http://murcia-nosevende.blogspot.com.es/

17 Desde la primera ley de extranjería, en 1985, en España se han promulgado un total de cinco leyes: LOEX 4/2000, LOEX 8/2000, LOEX 14/2003 y LOEX 2/2009.

18 http://www.laverdad.es/murcia/201605/23/revuelta-jornaleros-20160522003310.html

\section{Bibliografía}

Arcas, J., García, M., Hernández, I., Martínez, I., Olmedo, A., Montes, J. y Sabater, R. (2014). Análisis del sistema de ciencia, tecnología e innovación agroalimentaria en la Región de Murcia. Cuadernos de Estudios Agroalimentarios, vol.6, 171-204.

Bouvier, P. (1986). Anthropologie industrielle et culture ouvrière. Anthropologie et Sociétés, vol. 10, 1, 163169.

Camarero, L. (en prensa). Territorios encadenados, tránsitos migratorios y ruralidades adaptativas. Mundo Agrario

Carrasco Molina, J. (2008). Nicolas Gómez Tornero: una figura emblemática. http://josesimeoncarrasco.blogspot.com.es/2008/01/nicols-gmez-tornerouna-figura.html

Castaño Molina, M. A. (2012). Mujeres Trabajadoras: las abaraneras de los años cuarenta. Actas II Jornadas de Investigación y Divulgación sobre Abarán y el Valle de Ricote, 20 y 27 abril, 341-355. 
Castellanos, M. L. y Pedreño, A. (2001). Desde el Ejido al accidente de Lorca. Las amargas cosechas de los trabajadores inmigrantes en los milagrosos vergeles de la agricultura mediterránea. Sociología del Trabajo, 42, 3-31.

Chen, H. (2011). The governance and evolution of local production networks in a cluster: the case of Taiwan’s machine tool industry. GeoJournal, 76 (6), 605-622.

Chesterton, G. K. (2010). Los límites de la cordura. El distributismo y la cuestión social. Madrid: El Buey Mudo

Coe, N. y Hess, M. (2013). Global Production Networks, labour and development. Geoforum, 2013, vol. 44, 4-9

Coe, N. y Hess, M. (2011). Local and Regional development. A GPN approach. En A. Pike, A. RodriguezPosse yJ.Tomaney (Eds.), Handbook of Local and Regional Development (pp. 128-140). London: Routledge.

Coe, N., Dicken, P. y Hess, M. (2008). Global production networks: realizing the potential. Journal of Economic Geography, 8(3), 271-295.

Corrado, A., de Castro, C. y Perrotta, D. (eds) (2017). Migration and Agriculture: Mobility and Change in the Mediterranean Area, London: Routledge

Cumbers, A. y McKinnon, D. (2011). Putting "the political" back into the region. Power, agency and reconstitued regional political economy. En A. Pike, A. Rodriguez-Posse \& J. Tomaney (Eds.), Handbook of Local and Regional Development (pp. 248-260). London: Routledge,

de Castro, C., Moraes, N. y Cutillas, I. (2017) Gobernar la producción y el trabajo por medio de estándares. El caso de la industria agroalimentaria en Murcia”. Política y Sociedad 54(1)

de Castro, C. (2014) La desdemocratización de las relaciones laborales en los enclaves globales de producción agrícola. En Pedreño, A. (coord.) De cadenas, migrantes y jornaleros. Los territorios rurales en las cadenas globales agroalimentarias, (pp. 59-77). Madrid, Talasa.

Dicken, P. (2011). Global Shift. Mapping the changing contours of the global economy. London: The Gildford Press.

DIRCE. (2015). Directorio Central de Empresas, INE http://www.ine.es/jaxi/menu.do? type=pcaxis\&path=/t37/p201/\&file=inebase [último acceso 29 de junio 2016]

Gadea, E. Pedreño, A., y de Castro, C. (2017) Producing and mobilizing vulnerable workers. The agribusiness of the Region of Murcia, Spain. En A. Corrado, C. de Castro, y D. Perrotta (eds). Migration and Agriculture: Mobility and Change in the Mediterranean Area (pp. 79-94). London: Routledge

Gereffi, G. (1994). The Organization of Buyer-Driven Global Commodity Chains: How U.S. Retailers Shape Overseas Production Networks. En G. Gereffi y M. Korzeniewicz (Eds.), Commodity chains and global capitalism (pp. 95-122). Westport CT: Praeger.

Gereffi, G., Humphrey, J., y Sturgeon, T. (2005). The governance of global value chains. Review of International Political Economy, 12, 78-104.

Glassman, J. (2011). The Geo-political Economy of Global Production Networks in Geography Compass, 5(4), 154-164

Gómez Sánchez, A. (2002). Estampas de una infancia. V Curso: Abarán, acercamiento a una realidad (pp.78-79). Abarán: Centro de Estudios Abaraneros. 
Harvey, D. (2007). Breve historia del neoliberalismo. Madrid: Akal

Hellio, E. (2016). 'They know that you'll leave, like a dog moving onto the next bin'. Undocumented male and seasonal contracted female workers in agricultural labour market of Huelva, Spain. En A. Corrado, C. de Castro \& D. Perrotta (Eds.), Migration and Agriculture: Mobility and Change in the Mediterranean Area (pp. 140-158). London: Routledge,

Henderson, J., Dicken, P., Hess, M., Coe, N. M., Yeung, H. W. (2002). Global production networks and the analysis of regional development. Review of International Political Economy, 9(3), 436-464.

INFO (2015). Informe Sectorial. El sector hortofrutícola en la Región de Murcia. Murcia: Instituto de Fomento de la Región de Murcia.

INFO (2016). Informe Sectorial. El sector hortofrutícola en la Región de Murcia. Murcia: Instituto de Fomento de la Región de Murcia.

Keating, M. (Ed.). (2004). Regions and Regionalism in Europe. En The International Library of Comparative Public Policy.. Cheltenham (Reino Unido): Edward Elgar.

Levy, D. (2008). Political Contestation in Global Production Networks. Academy of Management Review, 33(4), 943-963.

Logan, J. y Molotch, H.L. (1987). Urban Fortunes. The Political Economy of Place. Los Ángeles: University of California Press.

Manzanares, D. (2006). Determinación de los salarios de hombres y mujeres en la industria de conservas vegetales, 1939-1975. Trabajo: Revista andaluza de relaciones laborales, 17, 31-54.

Martin, R. (2015). Rebalancing the Spatial Economy the Challenge for Regional Theory. En Territory, Politics, Governance, 3(3), 235-272.

Martínez Carrión, J.M. (2002). Economía de la Región de Murcia. Murcia: Editora Regional de Murcia.

Martínez-Carrasco, F. y Martínez, J. M. (2011). El clúster agroalimentario de la Región de Murcia. Cuadernos de Estudios Agroalimentarios, vol.2, 175-198.

McMichael, P. (2009). A food regime genealogy. Journal of Peasant Studies, 36(1), 139-169.

Narotzky, S. (1988). Trabajar en familia. Mujeres, hogares y talleres. Madrid: Consejo Superior de Investigaciones Científicas

Palenzuela, P. (1995). Las culturas del trabajo. Una aproximación antropológica. Sociología del Trabajo, 24, 3-28.

Palenzuela, P. (2014). Culturas del trabajo e identidad local: pescadores y mineros en Québec. Sociología del trabajo, 81, 68-89.

Pedreño, A., Gadea, E. y García, A. (2013). Jornaleras de la globalización en el campo murciano. En M. J. Sánchez e I. Serra (Coords.), Ellas se van, Mujeres migrantes en Estados Unidos y España (pp. 220-237). México: Instituto de Investigaciones Sociales - UNAM.

Pedreño, A., Gadea, E. y de Castro, C. (2014) Labor, Gender and Political Conflicts in the Global Agri-food System. The case of Agri-export model of Murcia, Spain. In Bonanno, A. y Cavalcanti, J.S. (eds.) Labor Relations in a Globalized Food. (pp. 193-214). Bingley: Emerald,

Pérez Picazo, M. T. y Lemeunier, G. (1990). Agricultura y desarrollo regional en Murcia, 1750-1980. Revista Áreas, 12, 225-236. 
Pike, A., Rodríguez-Pose, A. y Tomaney, J. (2006). Desarrollo local y regional. Valencia: Publicaciones Universidad de Valencia

Pike, A., Rodríguez-Pose, A. y Tomaney, J. (2011), Introduction: A handbook of local and regional development. En A. Pike et al. (Eds.), Handbook of local and regional development (pp. 1-12). London: Routledge.

Pike, A., Rodríguez-Pose, A. y Tomaney, J. (2016). Shifting horizons in local and regional development. Regional Studies 51(1), pp. 46-57

Reigada, A. (2012). Más allá del discurso sobre la 'inmigración ordenada’: contratación en origen y feminización del trabajo en los campos de fresas. Política y Sociedad, 49(1), 103-122

Saglio, J. (1991). Intercambio social e identidad colectiva en los sistemas industriales. Sociología del trabajo, 1, 45-62.

Segura, P. y Pedreño, A. (2006) La hortofruticultura intensiva de la Región de Murcia: un modelo productivo diferenciado. En M. Etxezarreta (coord.) La agricultura española en la era de la globalización, (pp. 369421). Madrid: Servicio de Publicaciones del Ministerio de Agricultura, Pesca y Alimentación,.

Sierra, J. (1990). El obrero soñado. Ensayo sobre el paternalismo industrial. Asturias, 1860-1917. Madrid: Siglo Veintiuno.

Sola, J. (2014). La desregulación laboral en España, 1984-1997 (Tesis doctoral). Universidad Complutense de Madrid, Madrid.

Stone, C. (1989). Regime Politics: Governing Atlanta, 1946-1988. Kansas: University Press of Kansas.

Wallerstein, I. (2000). The Essential Wallerstein. New York: The New Press

Yeung, H. W. C. (2009). Regional Development and the Competitive Dynamics of Global Production Networks: An East Asian Perspective. Regional Studies, 43(3), 325-351.

Zhu, H. y He, Z. (2016). Global and local governance, industrial and geographical dynamics: A tale of two clusters. Environment and Planning C, 34 (8), pp. 1453-1473 